\title{
A survey Paper on Feature Extraction and Classification in ECG Biometric Signal
}

\author{
Kalyani U. Girde ${ }^{1}$, Asst. Prof. Nandini Dhole ${ }^{2}$ \\ Student (ME), Department of Electronics and Telecommunication, RMD Sinhgad School of Engineering, Pune, India ${ }^{1}$ \\ Department of Department of Electronics and Telecommunication, RMD Sinhgad School of Engineering, Pune, India ${ }^{2}$
}

\begin{abstract}
This Customary biometric recognition frameworks regularly utilize physiological attributes, for example, unique mark, confront, iris, and so forth. Later a long time have seen a developing enthusiasm for electrocardiogram (ECG) based biometric acknowledgment procedures, particularly in the field of clinical prescription. In existing ECG based biometric recognition techniques, feature extraction and classifier configuration are as a rule performed independently. In this paper, multitask learning approach is proposed in which highlight extraction and classifier configuration are completed all the while. Weights are allocated to the features inside the kernel of each task. We break down the network comprising of all the feature weights into sparse and low rank components. The meagre part decides the highlights that are important to distinguish every person, and the low rank segment decides the normal component subspace that is pertinent to distinguish every one of the subjects. A quick improvement calculation is produced which requires just the principal arrange data. The execution of the proposed approach is shown through tests utilizing the MIT-BIH Normal Sinus Rhythm database.
\end{abstract}

Keywords: normalized minimum distance, PC, Biometrics, ECG, classification, feature selection, multitask learning.

\section{INTRODUCTION}

Electrocardiogram is one of the best cardiovascular examinations accessible. It gives wealth of data to finding and treatment. It is the after effect of electrical potential produced by the muscles of the heart. Since decades ago ECG is possibly utilized as a biometric for individual confirmation. In security frameworks biometrics assumes essential part to build the security level. Biometrics is identification of an individual based on the physiological and/or behavioral characteristics. It is highly difficult to do falsification in case of biometric frameworks. Biometrics has an extremely old history. Numerous physiological and behavioral biometric elements, for example, fingerprint, palm veins, face recognition, DNA, palm print, hand geometry, iris recognition, retina etc. and behavioral parameters, for example typing rhythm, gait, signature etc. are in use. The composition mechanism and electrical activity of the human heart inherit uniqueness from the individuality of DNA. In this way alongside all these biometric instruments, electrocardiogram can likewise be utilized as device to recognize the people. ECG as a biometrics is first time utilized by Lena Biel. The advantage of utilizing ECG as a biometric is that ECG checks the aliveness of a man. Biometric recognition gives validation by recognizing every individual in view of the organic and physiological signal characteristics. A number of identification strategies have been researched in the most recent decades, utilizing physical features such as finger prints, face images and biological signal behavioral such as electrocardiograph (ECG). Analysis of ECG signals as a biological tool for individual recognition has become a dynamic research field in the previous two decades.

\section{MOTIVATION}

A. To recognizing a man in view of human's special qualities and characteristics.

B. Each individual has a one of a kind ECG design because of the interesting physical and geometrical structure of his/her heart and body that is the reason ECG is valuable for biometric acknowledgment.

\section{OBJECTIVES}

A. Current biometric recognition frameworks mostly utilize physiological qualities, for example, fingerprint, face, iris, etc.

B. A biometric recognition utilizing a single-lead ECG signal which can be easily acquired in many situations.

\section{LITERATURE SURVEY}

H. Silvaet. al. [1] "Clinical data privacy and customization via biometrics based on ECG signals" Client identity approval is especially relevant for applications whereinformation security is basic, for example, Healthcare Information Systems (HIS), where patient records protection and medical acts traceability is extremely important. Records insurance and medical acts Traceability is critical. Current ways to deal with the issue include biometric solutions, in any case, conventional modalities just permit transient confirmation; readers are generally settled to a static area, what's more, direct contact or nearness is required. In Healthcare Information Systems (HIS), solid verification of both patients and caregivers is currently an issue which presents inefficiencies and reduces the quality of care because of 
blunders coming about because of mis-recognizable proofGovernment agencies and institutions worldwide, have started to recur to bio metric modalities as a way of improving current practices; however traditional bio metric systems still present some limitations.

M. M. Tawfiket. al. [2] "Human identification using QT signal and QRS complex of the ECG" In this paper the likelihood of utilizing the ECG signal as a Biometric feature for human identification is researched. A test set of 550 lead I ECG follows recorded from 22 sound individuals at various circumstances are utilized to approve the framework. The proposed framework extracts special parts of the ECG signal beginning from the QRS complex to the finish of the $\mathrm{T}$ wave. Effective Biometrics gives the best approach to recognize people in view of a one of a kind physiological or behavioral characteristic of the individual person. For a long time outer physiological biometrics like fingerprint, Iris recognition and face recognition were utilized. In this paper a feasibility study has been done on the success of utilizing ECG as inside Biometric feature, A database consisting of 22 healthy persons was built containing an extensive variety of heart rate qualities and three techniques were created for approval, the review demonstrates that the heart rate fluctuation has the real impact on the security of the ECG signal as a biometric feature which was treated by two techniques. Also the QRS complex of the ECG signal is proven to be stable against Heart rate variability and advantageous to be utilized a solitary as a Biometric include.

Y. Wang et. al. [3] "Analysis of human electrocardiogram for biometric recognition"Biometric recognition gives verification by recognizing every individual in view of the biological and physiological signal characteristics. In this paper, another approach for programmed investigation of single lead ECG for human recognition is proposed and evaluated. Taking after the pre-preparing step, the ECG stream is partitioned into separate windows where each window includes single beat of ECG signal. After effective QRS detection, various temporal, amplitude and AR coefficients are extracted andutilized as a contribution to a classifier keeping in mind the end goal to distinguish the people. A biometric framework for programmed examination of a single lead electrocardiogram (ECG) for human identification is exhibited in this work. The principal phase of this framework consists of a band-pass Butterworth filter used to remove noise and other artifacts present in the raw ECG signal.

B. Krishnapuramet. al. [4] "A Bayesian Approach to Joint Feature Selection and Classifier Design" This paper receives a Bayesian way to deal with at the same time learn both an optimal nonlinear classifier and a subset of predictor variables (or features) that are mostoptimal nonlinear classifier and a subset of predictor variables (or features) that are most applicable to the classification task. The approach utilizes heavy-tailed priors to promote sparsity in the usage of both premise capacities and components; these priors go about as regularizes for the probability work that prizes great order on the preparation information. We derive an expectation-maximization (EM) algorithm to efficiently compute a maximum a posteriori (MAP) point estimate of the different parameters. The algorithm is an extension of recent stateof-the-art sparse Bayesian classifiers, which in turn can be seen as Bayesian counterparts of support vector machines. Experimental comparisons utilizing part classifiers exhibit both parsimonious feature selection and excellent classification accuracy on a range of synthetic and benchmark data sets.

T. Jebaraet. al. [5] "Multi-task feature and kernel selection for SVMs" We register a typical component choice or, on the other hand part determination arrangement for various support vector machines (SVMs) trained on different yet inter-related datasets. The strategy advantageous when multiple classification tasks and differently labeled datasets exist over a common input space. Distinctive datasets can commonly strengthen a typical decision of portrayal or significant components for their different classifiers. We determine a multi-task representation learning approach utilizing the most extreme entropy segregation formalism. The resulting convex algorithms keep the global solution properties of support vector machines. However, in addition to multiple SVM classification/regression parameters they also jointly estimate an optimal subset of features or optimal combination of kernels. Experiments are shown on standardized datasets.

B. Bakkeret. al. [6] "Task clustering and gating for Bayesian multitask learning" Modelling a collection of similar regression or classification tasks can be improved by making the tasks 'learnfrom each other'. In machine learning, this subject is approached through 'multitask learning', where paralleltasks are modelled as multiple outputs of the same network. This is mostly implementedthrough the mixed-effects linear model where a difference is made among 'fixed effects', which are thesame for all tasks, and 'random effects', which may vary between tasks. In the present article we will adopt aBayesian approach in which some of the model parameters are shared (the same for all tasks) and others moreloosely connected through a joint prior distribution that can be learned from the data. We seek in this way tocombine the best parts of both the statistical multilevel method and the neural network machinery. The standard assumption expressed in both methodologies is that each undertaking can gain similarly well from any other task. In this article we expand the model by permitting more separation in the similitudes between assignments. One such extension is to make the prior mean depend on higher-level task characteristics. More unsupervised clustering of tasks is acquired in the event that we go from a solitary Gaussian preceding a blend of Gaussians. This can be further generalized to a mixture of expert's architecture with the gates depending on task characteristics 
R. Hoekemaet. al. [7] "Geometrical aspects of the inter individual variability of multilead ECG recordings"The electrocardiogram (ECG) has been used as a clinical tool to assess or to support the diagnosis in a cardiac patient ever since it was first recorded in man by Waller and further developed by Einthoven. The electrocardiogram (ECG) as measured from healthy subjects demonstrates a significant inter individual fluctuation. This fluctuation is brought about by geometrical and by physiological components. In this review, the relative commitment of the geometrical components is assessed. What's more a technique went for remedying for these variables is portrayed.This paper indicates that a major part in the inter individual variability of the ECG should be attributed to geometrical factor specifying the electric volume conduction properties of the thorax. The method presented for correcting for these factors so far was found to work well in a model to model evaluation, but failed in the direct application to measured ECGs.

L. Biel et. al. [8] "ECG analysis: A new approach in human identification" ESOPHAGEAL manometry (EGM) is a non-invasive toolfor esophagus examination as part of esophageal motilitydisorder diagnosis. Typically, any abnormality in esophagealfunction manifests itself in pressure dynamics inside the esophagus. In this paper, we propose a new approach to the analysis and modelling of esophageal manometry (EGM) data to assistthe diagnosis of esophageal motility disorders in humans. The proposed approach combines three techniques, namely, wavelet decomposition (WD), nonlinear pulse detection technique (NPDT), and statistical pulse modelling. Specifically, WD is applied to thefiltering of the EGM data, which is contaminated with electrocardiography (ECG) artifacts. In this paper, we propose a new approach to analysis and modelling of EGM data for esophageal motility disorders diagnosis.The proposed approach includes filtering of ECG and respirationartifacts from EGM data, detection/extraction of diagnosticallyvaluable portions of the EGM recordings, and development of astatistical model for differentiation between normal and abnormal cases.

I. westonet.al [9]" feature selection for SVMs", in many superviselearning problem feature selection important for a variety of reason. Generalization performance, running time requirement and constraints and interpretational issues imposed by the problem itself. We introduced a method of feature selection for support vector machine.

\section{SYSTEM ARCHITECTURE}

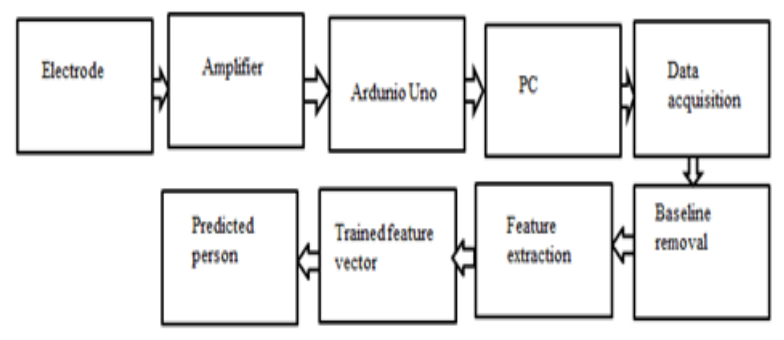

In the block diagram of fig 1 shows, Take ECG signal as input then use filter to remove the base line. The next block is the feature extraction. And then classify the signal by using normalized minimum distance. The basic feature extraction of ECG provides (amplitudes and intervals) to be used in succeeding automatic analysis. In recent times, a number of techniques have been proposed to detect these features.

Biometric technologies are one of the fast-developing fields of information security, gradually entering into all spheres of human activity. Today only three biometric methods have proved their efficiency. Namely, identification based on fingerprints, iris or retina, and face. Hand geometry, voice, writing and typing dynamics, etc. are also useful, depending on the purpose and range of application.

\section{CONCLUSION}

Another joint component extraction and classifier plan technique is proposed for single-lead ECG based biometric recognition. A non-fiducial feature is utilized. Feature extraction plays vital part in classification, it helps to increase classification accuracy. Feature extraction and classifier configuration are normally performed independently.In this paper, only focus on biometric recognition utilizing a single-lead ECG signal which can be effectively obtained by and large. Results on the MITBIH Normal Sinus Rhythm ECG database show the effectiveness of the proposed multitask learning approach over other conventional methodologies.

\section{REFERENCES}

[1] H. Silva, A. Lourenco, A. Fred, and J. Filipe, "Clinical data privacyand customization via biometrics based on ECG signals,"Lecture Notesin Computer Science, Information Quality in e-Health, vol. 7058, pp.121-132, 2011

[2] M. M. Tawfik and H. S. T. Kamal, "Human identification using QT signal and QRS complex of the ECG,"

[3] Y. Wang, F. Agrafioti, D. Hatzinakos, and K. N. Plataniotis, "Analysis ofhuman electrocardiogram for biometric recognition,"EURASIP Journalon Advances in Signal Processing, article 148658, pp. 1-11, 2008.

[4] B. Krishnapuram, A. J. Hartemink, L. Carin, and M. A. T. Figueiredo,"A Bayesian approach to joint feature selection and classifier design,"IEEE Transactions on Pattern Analysis and Machine Intelligence, vol.26, no. 9, pp. 1105-1111, 2004.

[5] T. Jebara, "Multi-task feature and kernel selection for SVMs," Proceedings of International Conference on Machine Learning (ICML), 2004.

[6] B. Bakker and T. Heskes, "Task clustering and gating for Bayesianmultitask learning,"Journal of Machine Learning Research, vol. 4, pp.83-99, 2003

[7] R. Hoekema, G. J. H. Uijen, and A. Van Oosterom, "Geometrical aspectsof the interindividual variability of multilead ECG recordings,'IEEETransactions on Biomedical Engineering, vol. 48, no. 5, pp. 551-559,2001

[8] L. Biel, O. Pettersson, L. Philipson, and P. Wide, "ECG analysis: A newapproach in human identification,"IEEE Transactions on Instrumenta-tion and Measurement, vol. 50, no. 3, pp. 808-812, 2001.

[9] J. Weston, S. Mukherjee, O. Chapelle, M. Pontil, T. Poggio, and V.Vapnik, "Feature Selection for SVMs,"Proceedings of Advances 
inNeural Information Processing Systems (NIPS), pp. 668-674, 2000

[10] R. Caruana, "Multitask learning," Ph.D. dissertation, School of Com-puter Science, Carnegie Mellon University, Pittsburgh, PA, 1997

\section{BIOGRAPHIES}

Ms. Kalyani U. Girde, currently studying in ME in VLSI and embedded system at RMD Sinhgad School of engineering Pune. The author has her personal field of interest in the domain of image processing, VLSI and embedded system.

Asst Prof. Nandini Dhole, currently working as Asst. Prof. at in RMD Sinhgad School of engineering pune. The author has her personal field of interest in the domain of image processing and digital signal processing. 